\title{
Evaluation of Oxidative Stress and Antioxidant Status in Diabetic and Hypertensive Women during Labor
}

\author{
Mashael M. Al-Shebly ${ }^{1}$ and Mahmoud A. Mansour ${ }^{2}$ \\ ${ }^{1}$ Department of Obstetrics and Gynecology, College of Medicine, King Saud University, P.O. Box 2925, Riyadh 11461, Saudi Arabia \\ ${ }^{2}$ Department of Pharmacology and Toxicology, College of Pharmacy, King Saud University, P.O. Box 2457, Riyadh 11451, Saudi Arabia
}

Correspondence should be addressed to Mahmoud A. Mansour, mansour1960us@yahoo.com

Received 5 February 2012; Revised 26 May 2012; Accepted 10 June 2012

Academic Editor: Martin-Ventura Jose Luis

Copyright (C) 2012 M. M. Al-Shebly and M. A. Mansour. This is an open access article distributed under the Creative Commons Attribution License, which permits unrestricted use, distribution, and reproduction in any medium, provided the original work is properly cited.

\begin{abstract}
Pregnancy in insulin-dependent diabetes mellitus is associated with a greater incidence of fetal abnormality. Animal studies suggested that increased free-radical production and antioxidant depletion may contribute to this risk. The objective of this work was to evaluate oxidative stress and antioxidant capacity in hypertensive, diabetics, and healthy control women during labor. Simultaneous determination of antioxidant enzymes activities, namely glutathione peroxidase (GSH-Px), glutathione reductase (GSH-red), superoxide dismutase (SOD), total antioxidant, and lipid peroxides measured as thiobarbituric acid-reactive substances (TBARS) levels, were carried out in maternal plasma during labor. Plasma GSH-Px activity was found to be significantly increased as it doubled in hypertensive, and diabetic women when compared with healthy control women $(P<0.05)$. In contrast, plasma SOD activity was significantly decreased in both groups when compared to the control group $(P<0.05)$. No significant differences were detected in GSH-Red activity between diabetic, hypertensive and control groups. Alterations in antioxidant enzyme activities were accompanied by a significant increase in the levels of plasma lipid peroxides in hypertensive and diabetic women during labor. Plasma levels of total antioxidants were significantly increased in diabetic women as compared with the control group. Based on our results, it may be concluded that enhanced generation of oxidative stress causes alteration of antioxidant capacity in diabetic and hypertensive women during labor. Alterations in antioxidant and prooxidant components may result in various complications including peroxidation of vital body molecules which may be regarded as an increased risk factor for pregnant women as well as the fetus.
\end{abstract}

\section{Introduction}

Lipid peroxidation is an oxidative process which occurs at low levels in all cells and tissues. Under normal conditions, a variety of antioxidant mechanisms serve to control this peroxidative process [1]. The generation of free radicals is a normal physiological process, but increased production of free radicals can act on lipids causing lipid peroxidation. The cells have evolved a number of counter acting antioxidant defenses. Free radical scavenging mechanisms includes enzymatic and nonenzymatic antioxidants which limit the cellular concentration of free radical and prevent excessive oxidative stress.

Pregnancy is a stressful condition in which many physiological and metabolic functions are altered to a considerable extent. Consequently, remarkable and dramatic events occur during this period [2]. Moreover, pregnancy is a physiological state, accompanied by a high-energy demand and an increased oxygen utilization, both of which may lead to increased oxidative stress. Oxidative stress occurs when there is an imbalance between free-radical production and the radical scavenging capacity of antioxidant systems [3]. Recently, Leal et al. [4] showed that there was a change in the prooxidant and antioxidant defenses that are inherent to pregnancy process.

There is evidence that both free-radical production and antioxidant defenses are disturbed in diabetes [5]. Therefore, it has been suggested over the last few years that oxidative stress in diabetes may be partly responsible for the development of diabetic complications [6]. The role of 
oxidative stress in the pathogenesis of insulin dependent diabetes mellitus has been implicated in several studies [7-9]. Increased lipid peroxidation products and altered antioxidative enzyme activities were also reported in noninsulin-dependent diabetes mellitus [10]. However, there are limited numbers of investigations in diabetic pregnancy [11-13]. Congenital malformations in diabetic pregnancies may be regarded in this context as a complication of maternal disease. Exposure of the developing rat embryo to free-radical generating systems in vitro will cause fetal abnormalities [14], and an increased oxygen tension is also associated with abnormal neural fold and crest development [15].

Little information is available regarding the early human development of antioxidant defenses, but the fetus is likely to be particularly sensitive to free-radical damage early in development when the major organ systems are developing. Therefore, it is likely that antioxidant status is especially important during this period.

In the present study, our aim was to investigate oxidative stress as an indicator of oxygen radical activity and antioxidant defenses in diabetic and hypertensive women and to compare the results with those obtained for healthy control women during labor.

\section{Results}

2.1. Blood Parameters. The blood parameters of all the three groups are summarized in Table 1 . Our results show that all blood parameters including RBCs count, WBCs count, platelets counts, and $\mathrm{HB}$ concentration in control, diabetic, and hypertensive women during labor were found to be statistically the same.

2.2. Plasma Concentrations of Urea, Creatinine, and Fasting Blood Glucose. Table 2 represents kidney function tests and fasting blood sugar. Plasma urea and creatinine concentrations were found to be significantly higher during labor in hypertensive women as compared to the control group concentrations. As expected, fasting blood sugar was significantly higher in diabetic women.

\subsection{Enzymatic Antioxidant Parameters and Oxidative Stress} Parameters. The antioxidant enzyme activities of all the three groups are summarized in Table 3. Plasma SOD levels of diabetic and hypertensive women during labor were significantly low as compared with the control group. In contrast, plasma GSH-Px levels of diabetic and hypertensive women were found to be significantly higher as compared to the control group. In addition, our results show that plasma GSH-red activity during labor in control, diabetic, and hypertensive women was found to be statistically the same.

Plasma levels of total antioxidants of diabetic women were found to be significantly higher than the respective control group, while there was no difference between control and hypertensive women.
Oxidative stress parameters of all the three groups are summarized in Table 4. Results show that the level of LPO of diabetic and hypertensive women during labor was found to be significantly higher than the control women.

\section{Discussion}

Pregnancy is a physiological state accompanied by a high energy demand and an increased oxygen requirement. Various compensatory adaptive changes, including increased ventilation for enhanced oxygen demand, occur with advancing pregnancy to meet the increasing requirements for proper bodily functions of the mother to fulfill the needs of the fetus [16]. Such a condition may be responsible for raised oxidative stress in pregnancy. The hypothesis underpinning this study was that impaired antioxidant status in diabetic and hypertensive women during labor might contribute to an increased risk of fetal abnormality. As outlined earlier, substantial evidence from animal models suggested that increased oxidative stress in the developing embryo is an important cause of abnormality, and that this can be prevented by antioxidants [17].

Our aim in the present study was to show the possibility of using the measured parameters as indicators of oxidative stress and antioxidant status during labor in diabetic, hypertensive, and healthy control women. In previous studies of antioxidant status in diabetics, abnormalities have usually been reported in the presence of diabetic complications or poor metabolic control. Indeed, in several studies antioxidant status and markers of lipid peroxidation have been normal in well-controlled diabetic subjects with no evidence of micro- or macroangiopathic complications $[18,19]$.

In the present study, we observed an increased level of plasma lipid peroxidation products (LPO) during labor in diabetic and hypertensive women. This may be attributed to over production of reactive oxygen species (ROS) or a deficiency of antioxidant defense. The results of the present study are in harmony with previous studies by Orhan et al. [20] and Tiwari et al. [21] who reported that the significant increase in plasma LPO in diabetic and anemic pregnant women, respectively, were parallel with a depletion of antioxidant enzymes as these enzymes are the major defense system of cells in normal aerobic reactions [22]

Similarly, during labor, plasma GSH-Px activity in diabetic and hypertensive women was increased significantly when compared to control women. This finding was in accordance with previous reports $[23,24]$ and the finding of Orhan et al. [20] who reported that erythrocyte GSH-Px activity was found to be significantly increased in hypertensive preeclamptic pregnancy and in insulindependent diabetic pregnancy. However, there are a number of conflicting reports as well $[25,26]$. It can be assumed that at low levels of oxidants the enzyme is deactivated, but after a certain higher level it gets activated by same oxidant(s). Therefore, an increase in GSH-Px activity coupled with an increase in plasma lipid peroxides in the same groups can be interpreted as a compensatory mechanism of the enzyme in order to quench the increased levels of hydrogen 
TABLE 1: Hemoglobin concentration, RBCs, WBCs, and platelets counts in control, diabetic, and hypertensive women during labor. Results are expressed as mean $\pm \mathrm{SE}$.

\begin{tabular}{lcccc}
\hline \multirow{2}{*}{ Groups } & HB & RBCs & WBCs & Platelet \\
& $\mathrm{g} / \mathrm{L}$ & $10^{12} / \mathrm{L}$ & $10^{9} / \mathrm{L}$ & $10^{9} / \mathrm{L}$ \\
\hline Control & $11.8 \pm 0.4$ & $4.17 \pm 0.1$ & $10.2 \pm 0.76$ & $229 \pm 11.2$ \\
Diabetic & $10.9 \pm 0.2$ & $3.88 \pm 0.079$ & $10.8 \pm 0.26$ & $237 \pm 15$ \\
Hypertensive & $10.9 \pm 1.1$ & $3.7 \pm 0.44$ & $10.4 \pm 2.6$ & $247 \pm 51$ \\
\hline
\end{tabular}

TABLE 2: Plasma urea, creatinine, and fasting blood glucose concentration in control, diabetic, and hypertensive women during labor.

\begin{tabular}{|c|c|c|c|}
\hline Groups & $\begin{array}{c}\text { Urea } \\
\mathrm{mmol} / \mathrm{L}\end{array}$ & $\begin{array}{c}\text { Creatinine } \\
\mu \mathrm{mol} / \mathrm{L}\end{array}$ & $\begin{array}{c}\text { Fasting blood sugar } \\
\mathrm{mmol} / \mathrm{L}\end{array}$ \\
\hline Control & $2.5 \pm 0.17$ & $47.6 \pm 3.1$ & $4.4 \pm 0.14$ \\
\hline Diabetic & $2.5 \pm 0.15$ & $46.2 \pm 1.6$ & $5.7 \pm 0.38^{*}$ \\
\hline Hypertensive & $3.77 \pm 0.65^{*}$ & $61.1 \pm 5.1^{*}$ & $5 \pm 0.4$ \\
\hline
\end{tabular}

Results are expressed as mean \pm SE.

* Significant difference from control group.

$P<0.05$.

peroxide. Therefore, elevated levels of GSH-Px activity in hypertensive and diabetic women during labor may be linked to increased oxidative stress, because it is well known that ROS, especially hydrogen peroxide stimulate GSH-Px activity. This is an expected outcome during the delivery of a fetus, since the high oxygen challenge occurring at birth might lead to increased formation of reactive oxygen species and subsequently hydrogen peroxide.

Our present data show that there was no statistically significant difference in plasma GSH-Red activity during labor in diabetic, hypertensive, and control women. However, Miranda Guisado et al. [27] recently clarified glutathione redox cycle in hypertensive disorders of pregnancy and found a significant decrease in its reduced form GSH with a parallel increase in the oxidized form GSSG and an increment in both GSH-Px and GSH-Red activities. In our study, we observed that the plasma level of GSH-red activity in hypertensive group is higher than control group but the difference did not reach significant level. The reason for the discrepancy may be due to difference between activity level of GSH-Red in RBCs and plasma or may be explained by the physiological properties of the enzyme GSH-red. Moreover the pentose phosphate pathway may not be perturbed, as the availability of NADPH, a cofactor for GSH-red functioning be may still sufficient.

Glutathione is a major intracellular antioxidant. Glutathione and other thiols maintain the redox balance of cells, thereby preventing oxidative damage. GSH-Px catalyzes the oxidation of GSH to GSSG and reduces hydrogen peroxides $\left(\mathrm{H}_{2} \mathrm{O}_{2}\right)$ to water. To maintain the balance between GSH and GSSG, the oxidized form of glutathione (GSSG) is reduced to GSH by GSH-red. For our future research, further assessment of GSH/GSSG ratio and hydrogen peroxides $\left(\mathrm{H}_{2} \mathrm{O}_{2}\right)$ could give us more information about oxidant and antioxidant status in diabetic and hypertensive women during labor. Therefore, we will take that in our consideration and it will be verified in the erythrocytes and plasma of diabetic and hypertensive women during labor.
Plasma SOD activity of diabetic and hypertensive women during labor was significantly lower than its corresponding level in control women although erythrocytes possess highly efficient antioxidant enzymes, such as SOD and GSH-Px compared to other cell types [28]. In the present study there were no differences in RBCs, WBCs, and platelet counts, and $\mathrm{HB}$ concentrations in different groups during labor. However, our results showed that diabetic and hypertensive women have lower SOD and higher GSH-Px activities than healthy control. Our results are in accordance with an earlier report [29]. The observed reduction in SOD activity in hypertensive and diabetic women to that of normal control women could be associated with deleterious effect of hypertension and diabetes, as the superoxide anion that is being generated continuously by numerous sources throughout the body would not be inactivated effectively and lead to an increase in its concentration. Enhanced generation of superoxide anion would result in greater oxidative stress and lipid peroxidation. Decreased SOD activity during labor in diabetic, and hypertensive groups may be linked to increased hydrogen peroxide since it is well known that ROS, especially superoxide anion and hydrogen peroxide $\left(\mathrm{H}_{2} \mathrm{O}_{2}\right)$, inhibit SOD activity [30]. SOD is a metalloprotein and accomplishes its antioxidant function by enzymatically detoxifying the peroxides $(-\mathrm{OOH})$ and $\mathrm{O}_{2}{ }^{--}$, respectively.

Plasma total antioxidants were significantly higher only in diabetic women as compared with the control group. Clinical biochemical parameters, such as urea and creatinine, were higher only in the hypertensive group when compared to the control. The significantly high level of urea and creatinine of these patients seem to be a result of a renal damage due to hypertension. On the other hand, plasma levels of fasting blood sugar were higher in diabetic women as compared with normal controls.

The present study shows elevated oxidative stress/lipid peroxidation in diabetic and hypertensive women during labor. Besides, SOD, GSH-Px, and total antioxidants seem to be appropriate biomarkers reflecting the status of antioxidant 
TABle 3: Plasma antioxidant enzymes activities and total antioxidant in control, diabetic, and hypertensive women during labor.

\begin{tabular}{|c|c|c|c|c|}
\hline Groups & $\begin{array}{l}\text { SOD } \\
\mathrm{U} / \mathrm{mL}\end{array}$ & $\begin{array}{c}\text { GSH-Px } \\
\text { U/L }\end{array}$ & $\begin{array}{c}\text { GSH-red } \\
\text { U/L }\end{array}$ & $\begin{array}{c}\text { Total antioxidant } \\
\mathrm{mmol} / \mathrm{L}\end{array}$ \\
\hline Control & $49.76 \pm 1.8$ & $35.6 \pm 3.4$ & $208 \pm 11.8$ & $0.59 \pm 0.04$ \\
\hline Diabetic & $32.76 \pm 2.4^{*}$ & $60.7 \pm 9.6^{*}$ & $194 \pm 13.3$ & $1.5 \pm 0.38^{*}$ \\
\hline Hypertensive & $32 \pm 3^{*}$ & $69.3 \pm 12.8^{*}$ & $253.5 \pm 49.7$ & $1 \pm 0.14$ \\
\hline
\end{tabular}

Results are expressed as mean \pm SE.

* Significant difference from control group.

$P<0.05$.

TABle 4: Plasma lipid peroxides measured as in control, diabetic, and hypertensive women during labor.

\begin{tabular}{lc}
\hline Groups & MDA $(\mathrm{nmol} / \mathrm{mL})$ \\
\hline Control & $2.3 \pm 0.47$ \\
Diabetic & $3.34 \pm 0.28^{*}$ \\
Hypertensive & $3.1 \pm 0.33^{*}$ \\
\hline
\end{tabular}

Results are expressed as mean \pm SE.

* Significant difference from control group.

$P<0.05$.

capacity in these diseases. The validation of these biomarkers for monitoring the efficiency of antioxidant supplementation during pregnancy should be further investigated. This supplementation may provide the prevention and/or attenuation of oxidative stress and enhancement of antioxidant status.

\section{Material and Methods}

4.1. Chemicals. All chemicals used in this study were of analytical grade.

4.2. Subjects. The present study was comprised of 62 pregnant women [namely control (24), diabetic (27) and hypertensive (11)] aged between 20-40 years. The subjects were selected amongst those attending the Department of Obstetrics and Gynaecology, King Khaled Hospital, King Saud University, Riyadh, Saudi Arabia. They were divided into groups of healthy controls, hypertensive, and insulindependent diabetic subject. Hypertension was defined according to the criteria of the Committee on Terminology of the American College of Obstetricians and Gynecologists, which has defined hypertension as a blood pressure greater than 140/90 $\mathrm{mmHg}$ before pregnancy or before 20 weeks of gestation. We defined insulin dependent diabetics diagnosed when not pregnant as Type I diabetes mellitus according to the National Diabetes Data Group Classification [31]. The diagnosis of the disease was made when two or more of the following plasma glucose concentrations were met or exceeded: fasting, $105 \mathrm{mg} / \mathrm{dL} ; 1 \mathrm{~h}, 190 \mathrm{mg} / \mathrm{dL} ; 2 \mathrm{~h}$, $165 \mathrm{mg} / \mathrm{dL}$; and $3 \mathrm{~h}, 145 \mathrm{mg} / \mathrm{dL}$.

All groups were non-alcoholic and non-smoking subjects having no history of diseases such as malignancy, heart disease, or having infections such as tuberculosis and HIV. Informed consent was obtained from each subject. The present study was approved by the Institutional Ethical Committee of King Saud University, Riyadh, Saudi Arabia.

4.3. Sample Collection. Venous blood $(6 \mathrm{~mL})$ was taken from each subject at the time of delivery and divided into three aliquots. Blood $(2 \mathrm{~mL})$ was transferred to an EDTA containing evacuated tube and was used to determine hemoglobin $(\mathrm{Hb})$, red blood cells (RBC), white blood cells (WBC), and platelets counts. $2 \mathrm{~mL}$ of whole blood was also transferred into a heparin containing tube and then centrifuged, plasma was separated and used for the estimation of lipid peroxide levels (LPO), antioxidant enzymes, namely superoxide dismutase (SOD), glutathione peroxidase (GSH$\mathrm{Px}$ ), glutathione reductase (GSH-red), and total antioxidant. The remaining $2 \mathrm{~mL}$ of venous blood was also centrifuged at $3000 \mathrm{rpm}$ for $15 \mathrm{~min}$, serum separated and used for the estimation of urea, creatinine and glucose.

\subsection{Biochemical Estimation}

4.4.1. Blood Cell Counting. Blood haemoglobin was determined by using the cyanomethemoglobin method [32]. Red blood cell, white blood cells and platelets counts were determined by using the Sysmax A-380 automated cell counter.

4.4.2. Measurement of the Antioxidant Enzyme Activities. Plasma SOD activity was determined as previously described by McCord and Fridovich [33] in a kinetic assay at $37^{\circ} \mathrm{C}$ using a test reagent kit for SOD (RANSOD-Randox, UK). The absorbance was measured at $505 \mathrm{~nm}$ and the results were expressed as $\mathrm{U} / \mathrm{mL}$.

GSH-Px activity was estimated in the plasma according to Paglia and Valentine [34] by a kinetic assay at $37^{\circ} \mathrm{C}$ using a test reagent kit (RANSEL-Randox, UK). The absorbance was measured at $340 \mathrm{~nm}$ and the results were expressed as U/L. The GSH-red was assayed by the method of Hazelton and Lang [35]. The absorbance was measured at $340 \mathrm{~nm}$ and the results were expressed as U/L. Total antioxidant was measured according to Miller et al. [36]. The absorbance was measured at $600 \mathrm{~nm}$ and the results were expressed as $\mathrm{mmol} / \mathrm{L}$.

4.4.3. Estimation of Lipid Peroxidation. Plasma levels of lipid peroxides were determined as TBARS and calculated as malondialdehyde (MDA) according to the method of 
Ohkawa et al. [37]. The absorbance was measured at $532 \mathrm{~nm}$ and the concentrations were expressed as nmol MDA/mL.

4.5. Statistical Analysis. Results are expressed as mean \pm SEM. Differences between obtained values were carried out by one way analysis of variance (ANOVA) followed by the Tukey-Kramer multiple comparison test. A $P$ value of 0.05 or less was considered as a statistically significant difference.

\section{Abbreviations}

ROS: $\quad$ Reactive oxygen species

SOD: $\quad$ Superoxide dismutase

GSH-Px: Glutathione peroxidase

GSH-Red: Glutathione reductase

LP: $\quad$ Lipid peroxide.

\section{Acknowledgment}

The present work was supported by operating grant from Research Center, College of Medicine, King Saud University (CMRC130146).

\section{References}

[1] H. Sies, "Oxidative stress: oxidants and antioxidants," American Journal of Medicine C, vol. 91, no. 3, 1991.

[2] S. Qanungo and M. Mukherjea, "Ontogenic profile of some antioxidants and lipid peroxidation in human placental and fetal tissues," Molecular and Cellular Biochemistry, vol. 215, no. 1-2, pp. 11-19, 2000.

[3] E. Granot and R. Kohen, "Oxidative stress in childhood-in health and disease states," Clinical Nutrition, vol. 23, no. 1, pp. 3-11, 2004.

[4] C. A. M. Leal, M. R. C. Schetinger, D. B. R. Leal et al., "Oxidative stress and antioxidant defenses in pregnant women," Redox Report, vol. 16, no. 6, pp. 230-236, 2011.

[5] T. J. Lyons, "Oxidized low density lipoproteins: a role in the pathogenesis of atherosclerosis in diabetes?" Diabetic Medicine, vol. 8, no. 5, pp. 411-419, 1991.

[6] J. W. Baynes, "Role of oxidative stress in development of complications in diabetes," Diabetes, vol. 40, no. 4, pp. 405412, 1991.

[7] S. K. Jain, "Hyperglycemia can cause membrane lipid peroxidation and osmotic fragility in human red blood cells," Journal of Biological Chemistry, vol. 264, no. 35, pp. 21340-21345, 1989.

[8] H. Orhan and G. Sahin, "Erythrocyte glutathione Stransferase activity in diabetes mellitus: the effect of the treatment," Fabad Journal of Pharmaceutical Sciences, vol. 24, no. 3, pp. 127-131, 1999.

[9] Y. Sato, N. Hotta, and N. Sakamoto, "Lipid peroxide level in plasma of diabetic patients," Biochemical Medicine, vol. 21, no. 1, pp. 104-107, 1979.

[10] H. Kaji, M. Kurasaki, and K. Ito, "Increased lipoperoxide value and glutathione peroxidase activity in blood plasma of type 2 (non-insulin-dependent) diabetic women," Klinische Wochenschrift, vol. 63, no. 16, pp. 765-768, 1985.

[11] D. Carone, G. Loverro, P. Greco, F. Capuano, and L. Selvaggi, "Lipid peroxidation products and antioxidant enzymes in red blood cells during normal and diabetic pregnancy," European
Journal of Obstetrics Gynecology and Reproductive Biology, vol. 51, no. 2, pp. 103-109, 1993.

[12] U. Kamath, G. Rao, C. Raghothama, L. Rai, and P. Rao, "Erythrocyte indicators of oxidative stress in gestational diabetes," Acta Paediatrica, vol. 87, no. 6, pp. 676-679, 1998.

[13] A. Loven, Y. Romem, I. Z. Pelly, G. Holcberg, and G. Agam, "Copper metabolism-a factor in gestational diabetes?" Clinica Chimica Acta, vol. 213, no. 1-3, pp. 51-59, 1992.

[14] P. C. Jenkinson, D. Anderson, and S. D. Gangolli, "Malformations induced in cultured rat embryos by enzymically generated active oxygen species," Teratogenesis Carcinogenesis and Mutagenesis, vol. 6, no. 6, pp. 547-554, 1986.

[15] G. M. Morriss and D. A. T. New, "Effect of oxygen concentration on morphogenesis of cranial neural folds and neural crest in cultured rat embryos," Journal of Embryology and Experimental Morphology, vol. 54, pp. 17-35, 1979.

[16] E. Gitto, R. J. Reiter, M. Karbownik et al., "Causes of oxidative stress in the pre- and perinatal period," Biology of the Neonate, vol. 81, no. 3, pp. 146-157, 2002.

[17] U. J. Eriksson and L. A. H. Borg, "Protection by free oxygen radical scavenging enzymes against glucose-induced embryonic malformations in vitro," Diabetologia, vol. 34, no. 5, pp. 325-331, 1991.

[18] P. E. Jennings and A. H. Barnett, "New approaches to the pathogenesis and treatment of diabetic microangiopathy," Diabetic Medicine, vol. 5, no. 2, pp. 111-117, 1988.

[19] S. V. McLennan, S. Heffernan, L. Wright et al., "Changes in hepatic glutathione metabolism in diabetes," Diabetes, vol. 40, no. 3, pp. 344-348, 1991.

[20] H. Orhan, L. Önderoglu, A. Yücel, and G. Sahin, "Circulating biomarkers of oxidative stress in complicated pregnancies," Archives of Gynecology and Obstetrics, vol. 267, no. 4, pp. 189195, 2003.

[21] A. K. M. Tiwari, A. A. Mahdi, F. Zahra, S. Chandyan, V. K. Srivastava, and M. P. S. Negi, "Evaluation of oxidative stress and antioxidant status in pregnant anemic women," Indian Journal of Clinical Biochemistry, vol. 25, no. 4, pp. 411-418, 2010.

[22] H. D. Scheibmeir, K. Christensen, S. H. Whitaker, J. Jegaethesan, R. Clancy, and J. D. Pierce, "A review of free radicals and antioxidants for critical care nurses," Intensive and Critical Care Nursing, vol. 21, no. 1, pp. 24-28, 2005.

[23] J. T. Uotila, R. J. Tuimala, T. M. Aarnio, K. A. Pyykko, and M. O. Ahotupa, "Findings on lipid peroxidation and antioxidant function in hypertensive complications of pregnancy," British Journal of Obstetrics and Gynaecology, vol. 100, no. 3, pp. 270276, 1993.

[24] J. Uotila, R. Tuimala, and K. Pyykko, "Erythrocyte glutathione peroxidase activity in hypertensive complications of pregnancy," Gynecologic and Obstetric Investigation, vol. 29, no. 4, pp. 259-262, 1990.

[25] C. A. Hubel, J. M. Roberts, R. N. Taylor, T. J. Musci, G. M. Rogers, and M. K. McLaughlin, "Lipid peroxidation in pregnancy: new perspectives on preeclampsia," American Journal of Obstetrics and Gynecology, vol. 161, no. 4, pp. 10251034, 1989.

[26] D. Wickens, M. H. Wilkins, and J. Lunec, "Free-radical oxidation (peroxidation) products in plasma in normal and abnormal pregnancy," Annals of Clinical Biochemistry, vol. 18, no. 3, pp. 158-162, 1981.

[27] M. L. Miranda Guisado, A. J. Vallejo-Vaz, P. Stiefel García Junco et al., "Abnormal levels of antioxidant defenses in a large sample of patients with hypertensive disorders of pregnancy," Hypertension Research, vol. 35, no. 3, pp. 274-278, 2012. 
[28] A. Kumerova, A. Lece, A. Skesters, A. Silova, and V. Petuhovs, "Anaemia and antioxidant defence of the red blood cells," Materia Medica Polona, vol. 30, no. 1-2, pp. 12-15, 1998.

[29] E. Kurtoglu, A. Ugur, A. K. Baltaci, and L. Undar, "Effect of iron supplementation on oxidative stress and antioxidant status in iron-deficiency anemia," Biological Trace Element Research, vol. 96, no. 1-3, pp. 117-123, 2003.

[30] M. Isler, N. Delibas, M. Guclu et al., "Superoxide dismutase and glutathione peroxidase in erythrocytes of patients with iron deficiency anemia: effects of different treatment modalities," Croatian Medical Journal, vol. 43, no. 1, pp. 16-19, 2002.

[31] National Diabetes Data Group, "Classification and diagnosis of diabetes mellitus and other categories of glucose intolerance," Diabetes, vol. 28, no. 12, pp. 1039-1057, 1979.

[32] International Nutritional Anemia Consultative Group, Measurements of Iron Status, INACG, Washington, DC, USA, 1985.

[33] J. M. McCord and I. Fridovich, "Superoxide dismutase. An enzymic function for erythrocuprein (hemocuprein)," Journal of Biological Chemistry, vol. 244, no. 22, pp. 6049-6055, 1969.

[34] D. E. Paglia and W. N. Valentine, "Studies on the quantitative and qualitative characterization of erythrocyte glutathione peroxidase," The Journal of Laboratory and Clinical Medicine, vol. 70, no. 1, pp. 158-169, 1967.

[35] G. A. Hazelton and C. A. Lang, "Glutathione contents of tissues in the aging mouse," Biochemical Journal, vol. 188, no. 1, pp. 25-30, 1980.

[36] N. J. Miller, C. Rice-Evans, M. J. Davies, V. Gopinathan, and A. Milner, "A novel method for measuring antioxidant capacity and its application to monitoring the antioxidant status in premature neonates," Clinical Science, vol. 84, no. 4, pp. 407412,1993

[37] H. Ohkawa, N. Ohishi, and K. Yagi, "Assay for lipid peroxides in animal tissues by thiobarbituric acid reaction," Analytical Biochemistry, vol. 95, no. 2, pp. 351-358, 1979. 


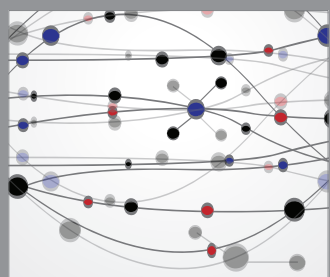

The Scientific World Journal
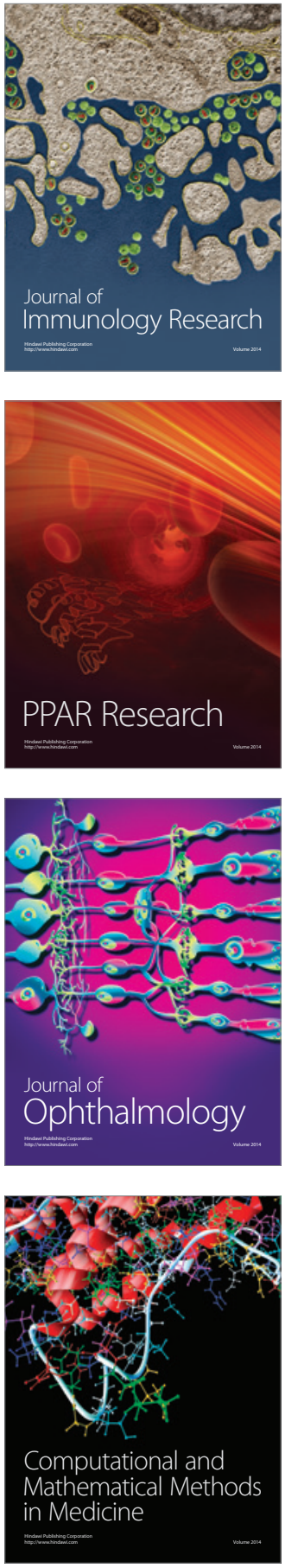

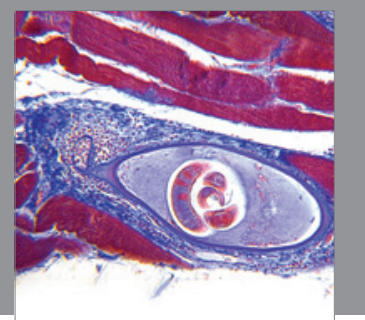

Gastroenterology

Research and Practice
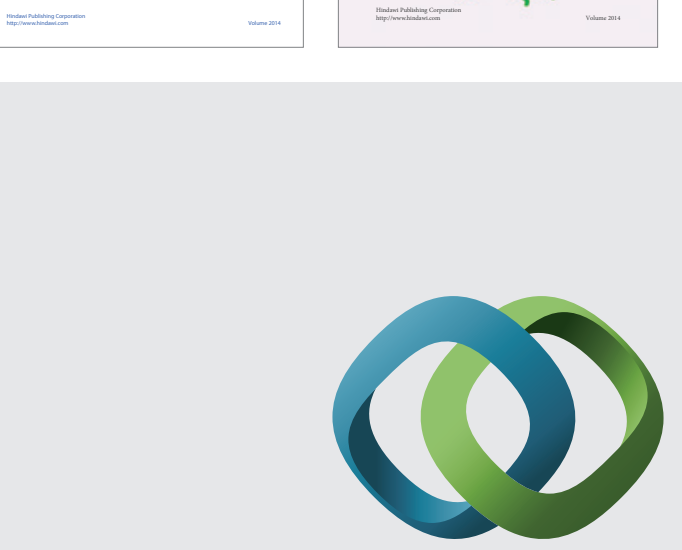

\section{Hindawi}

Submit your manuscripts at

http://www.hindawi.com
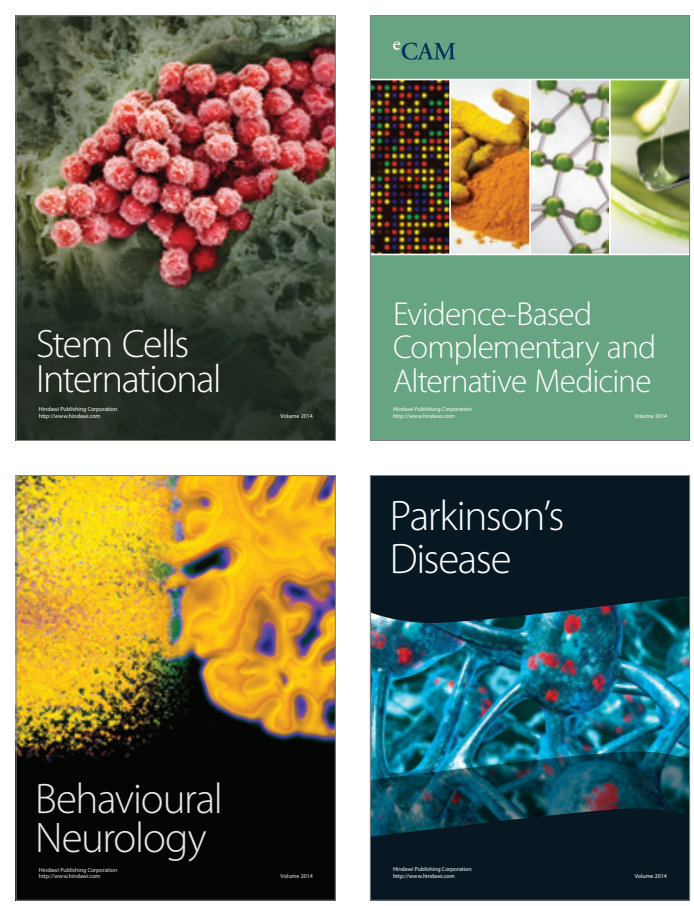

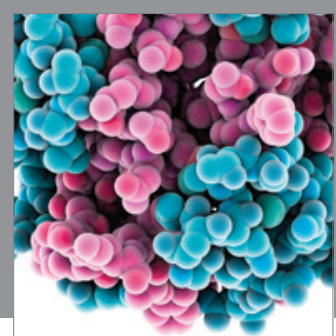

Journal of
Diabetes Research

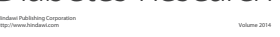

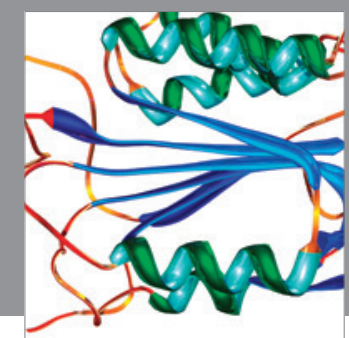

Disease Markers
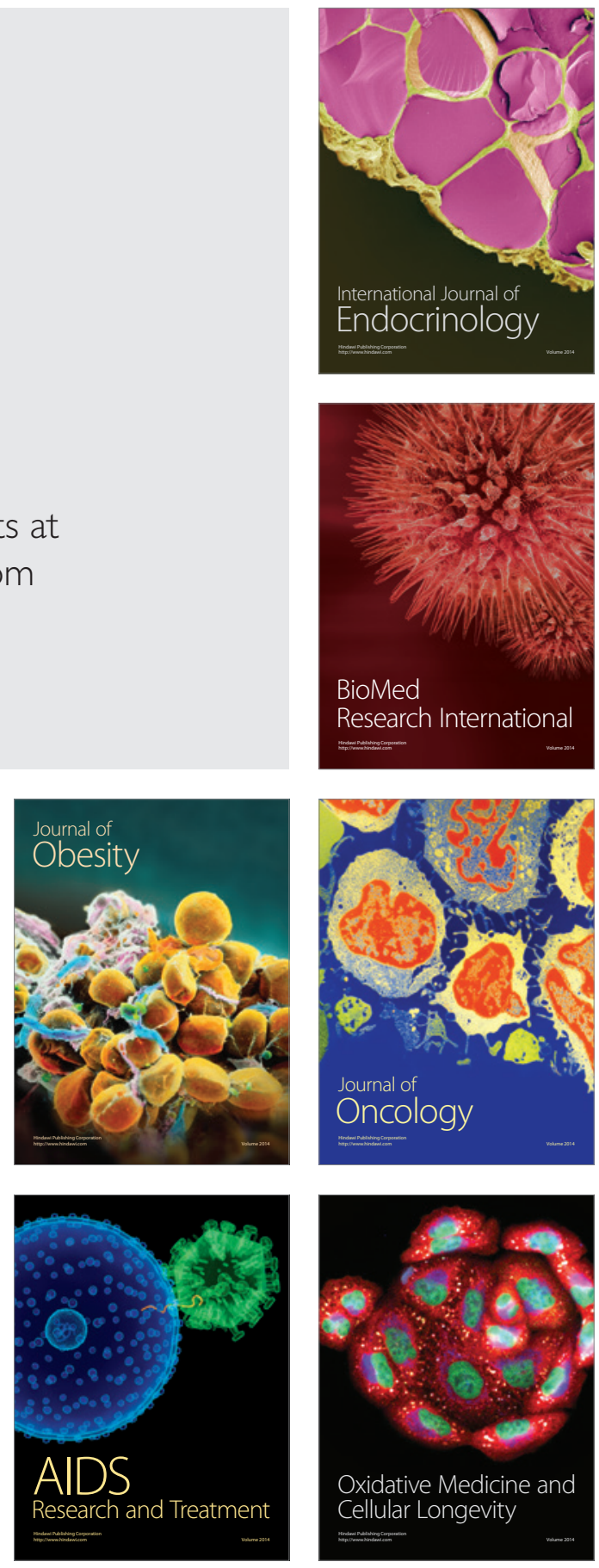